\title{
Measuring economic development of EU countries - a multivariate approach
}

\author{
Nemanja Milenković, Aleksandar Đoković, Nikola Cvetković \\ University of Belgrade, Faculty of Organizational Sciences
}

\begin{abstract}
The measure of a country's economic development is one of the most critical and highly debated issues in economic research. Different approaches have been applied and numerous indicators have been used in order to elaborate this multidimensional concept. The aim of this research is to present one synthesized indicator that can quantitatively point out any country's development level. In this paper, the statistical I-distance method is thoroughly explained and has been applied to $28 \mathrm{EU}$ countries. I-distance is metric distance in an $n$-dimensional space. It is created with the idea to avoid redundancy of the information in the process of ranking entities, based on different indicators for measuring intensity of the observed phenomena. Furthermore, stepwise I-distance procedure has been used in order to examine statistical significance of each development indicator. This procedure allows iterative exclusion of statistically insignificant indicators, whereupon the final results are obtained using the most important development indicators.
\end{abstract}

Keywords: I-distance method; indicators; multivariate analysis; ranking; stepwise method. 